\title{
Temperature transcends partner specificity in the symbiosis establishment of a cnidarian
}

\author{
Marcela Herrera $^{1} \cdot$ Shannon G. Klein ${ }^{2} \cdot$ Sara Campana ${ }^{1,3} \cdot$ Jit Ern Chen $\mathbb{1}^{1,4} \cdot$ Arun Prasanna ${ }^{1} \cdot$ Carlos M. Duarte $\mathbb{1}^{2} \cdot$ \\ Manuel Aranda $\mathbb{D}^{1}$
}

Received: 16 January 2020 / Revised: 13 August 2020 / Accepted: 2 September 2020 / Published online: 15 September 2020

(c) The Author(s) 2020. This article is published with open access

\begin{abstract}
Coral reef research has predominantly focused on the effect of temperature on the breakdown of coral-dinoflagellate symbioses. However, less is known about how increasing temperature affects the establishment of new coral-dinoflagellate associations. Inter-partner specificity and environment-dependent colonization are two constraints proposed to limit the acquisition of more heat tolerant symbionts. Here, we investigated the symbiotic dynamics of various photosymbionts in different host genotypes under "optimal" and elevated temperature conditions. To do this, we inoculated symbiont-free polyps of the sea anemone Exaiptasia pallida originating from Hawaii (H2), North Carolina (CC7), and the Red Sea (RS) with the same mixture of native symbiont strains (Breviolum minutum, Symbiodinium linucheae, S. microadriaticum, and a Breviolum type from the Red Sea) at 25 and $32{ }^{\circ} \mathrm{C}$, and assessed their ITS2 composition, colonization rates, and PSII photochemical efficiency $(F v / F m)$. Symbiont communities across thermal conditions differed significantly for all hosts, suggesting that temperature rather than partner specificity had a stronger effect on symbiosis establishment. Overall, we detected higher abundances of more heat resistant Symbiodiniaceae types in the $32{ }^{\circ} \mathrm{C}$ treatments. Our data further showed that PSII photophysiology under elevated temperature improved with thermal pre-exposure (i.e., higher $F v / F m$ ), yet, this effect depended on host genotype and was influenced by active feeding as photochemical efficiency dropped in response to food deprivation. These findings highlight the role of temperature and partner fidelity in the establishment and performance of symbiosis and demonstrate the importance of heterotrophy for symbiotic cnidarians to endure and recover from stress.
\end{abstract}

Supplementary information The online version of this article (https:// doi.org/10.1038/s41396-020-00768-y) contains supplementary material, which is available to authorized users.

Manuel Aranda

manuel.aranda@kaust.edu.sa

1 Red Sea Research Center (RSRC), Biological and Environmental Sciences and Engineering Division (BESE), King Abdullah University of Science and Technology (KAUST), Thuwal, Saudi Arabia

2 Red Sea Research Center (RSRC) and Computational Bioscience Research Center (CBRC), Biological and Environmental Sciences and Engineering Division (BESE), King Abdullah University of Science and Technology (KAUST), Thuwal, Saudi Arabia

3 Present address: Institute for Biodiversity and Ecosystem Dynamics, Faculty of Science, University of Amsterdam, 1090 GE Amsterdam, The Netherlands

4 Present address: Department of Biological Sciences, School of Science and Technology, Sunway University, Subang Jaya, Selangor, Malaysia

\section{Introduction}

Heat-induced coral bleaching - the breakdown of a fragile partnership between the coral host and photosynthetic algae of the family Symbiodiniaceae [1] — is a dominant driver of coral reef degradation. Increase in sea surface temperatures by only $1-2{ }^{\circ} \mathrm{C}$ above mean summer maxima often causes mass mortality of corals and many other species that depend on the ecosystems these organisms build [2]. However, coral responses to heat stress are not uniform, but instead some species and genotypes resist and/or recover better than others, either because of host genetics [3-7], symbiont identity [8-12], and/or acclimation capacities of the holobiont [13-15]. Particularly, flexibility of the coral host to associate with various photosymbiont strains might be crucial for coping with rapid fluctuations in the environment [16]. Many species can, for example, improve their thermal resilience by temporarily changing their algal symbiont communities (i.e., "shuffling" of preexistent types and/or "switching" to new ones) before, during, and after heat stress events 
[17-19]. However, this is not a universal feature of coralSymbiodiniaceae symbioses $[20,21]$ given that some hosts and symbionts can be less flexible in their association than others. The onset and maintenance of a persistent symbiosis depends on the functional compatibility between the two (or more) partners [22-24] and environmental settings [25-28].

As climate change intensifies and oceans become warmer it becomes increasingly relevant to understand the effect of temperature on the establishment of coral symbiosis. Exposure to elevated temperatures can drastically reduce the initial uptake of symbionts [29] and even impair larvae from establishing symbiosis [30]. Thermal stress can also affect the ability of different taxa to colonize the host such that thermally sensitive symbionts have greater colonization success at lower temperatures and conversely, more heat tolerant types prevail in warmer environments [26, 31]. Given this, it is fair to assume that even if native symbionts adapt to increasing temperatures over time, climate warming might still promote symbioses with already existing heat resistant Symbiodiniaceae strains (e.g., Durusdinium sp. [8, 12, 32]) and as a result, change the structure and diversity of host-symbiont assemblages on reefs. Even so, a recent study showed that partner fidelity (i.e., the inherent compatibility between host and symbiont partners) can limit the acquisition of non-native, more thermotolerant symbionts, even under stress conditions [33]. Emerging evidence indicates that coral-Symbiodiniaceae specificity may be a genetically determined trait in some species [34]; and so host-microbe relationships can be highly conserved. This trait has also been documented for coral bacterial communities $[35,36]$. Thus, understanding symbiosis specificity is not only important to predict the adaptive evolution of a particular association but also the potential to form new ones, especially in light of future climate change scenarios.

Here, we used Exaiptasia pallida [37] (commonly known as Aiptasia) to investigate the extent to which symbiotic associations are host genotype- and temperature-dependent, and how this affects the stress resilience of cnidarian holobionts. We tested the specificity of several Symbiodiniaceae species exhibiting various degrees of heat tolerance $[12,32]$ in different hosts under "optimal" and elevated temperature conditions. The species were: Breviolum minitum which is native to H2 Aiptasia from Hawaii [38], Symbiodinium linucheae [39] from North Carolina and symbiotic with CC7 [40], and $S$. microadriaticum and a Breviolum strain, isolated from a Red Sea (RS) population [41]. These lineages (host and symbiont) originate from locations with distinct thermal profiles and thus, have likely acquired specific adaptations to their local environments, which is reflected in different stress responses of both partners. In particular, Aiptasia and Symbiodiniaceae taxa from the Red Sea and North Carolina are known to be more thermotolerant than those from Hawaii [39]. Importantly, we selected only symbiont types that are native to our model system (i.e., strains that are naturally found as main symbionts in Aiptasia) and so, we did not perform experiments with the exceptionally heat tolerant strain; D. trenchii $[8,12,32]$.

We inoculated symbiont-free polyps of all host lines with mixtures comprising equal proportions of the four symbiont strains mentioned above. We assessed the colonization dynamics and PSII photophysiology of these associations among thermal treatments. Based on previous studies $[29,33,42,43]$ in which hosts were simultaneously exposed to multiple symbiont types, we hypothesized that symbionts native to the host line will dominate under "optimal" conditions, but, as temperature increases, more heat tolerant (nonnative) types will prevail. In line with evidence showing that pre-exposure to stress improves resilience (reviewed in [44]), we further predicted that the thermal condition in which initial inoculations were performed would have an influence on the holobiont's response to subsequent elevated temperature. Specifically, we hypothesized that Aiptasia pre-exposed to heat would exhibit greater thermal tolerance than hosts from control, "optimal" conditions, regardless of their genotype and symbiont composition.

\section{Materials and methods}

\section{Experimental organisms}

Aiptasia from three different clonal laboratory strains were used in this study (see Introduction, Table 1). Briefly,
Table 1 Details of the Symbiodiniaceae cultures used to perform inoculations.

\begin{tabular}{|c|c|c|c|c|}
\hline $\begin{array}{l}\text { Symbiont } \\
\text { strain ID }\end{array}$ & $\begin{array}{l}\text { Aiptasia } \\
\text { host source }\end{array}$ & $\begin{array}{l}\text { Original geographic } \\
\text { location }\end{array}$ & Symbiodiniaceae species & $\begin{array}{l}\text { Majority } \\
\text { ITS2 sequence }\end{array}$ \\
\hline SSB01 & $\mathrm{H} 2$ & Hawaii, USA & Breviolum minutum & B1 \\
\hline SSA01 & $\mathrm{CC} 7$ & North Carolina, USA & Symbiodinium linucheae & A4 \\
\hline RS-A & $\mathrm{RS}$ & $\begin{array}{l}\text { Al Lith, Saudi Arabia } \\
\text { (central Red Sea) }\end{array}$ & $\begin{array}{l}\text { Symbiodinium } \\
\text { microadriaticum }\end{array}$ & A1 \\
\hline RS-B & $\mathrm{RS}$ & $\begin{array}{l}\text { Al Lith, Saudi Arabia } \\
\text { (central Red Sea) }\end{array}$ & Breviolum sp. & RS-B1* \\
\hline
\end{tabular}

*SymPortal revealed different ITS2 type profiles for the Breviolum strains from Hawaii and the Red Sea, thus these were classified as B1 and RS-B1, respectively. 
anemones were reared in clear polycarbonate containers ( 2 L capacity; Cambro Camwear, USA) filled with autoclaved natural seawater $(\sim 39 \mathrm{psu}$ and $\mathrm{pH} \sim 8)$ at $25^{\circ} \mathrm{C}$ under $\sim 40$ $\mu \mathrm{mol}$ photons $/ \mathrm{m}^{2} \mathrm{~s}$ white light on a $12: 12 \mathrm{~h}$ light:dark cycle (daytime of 06.00-18.00). These light levels, which are similar to those reported by other studies working with Aiptasia [36, 45-48], were chosen to support optimal growth of the animals but also because all symbiont strains used here perform well under this irradiance. Anemones were fed with freshly hatched Artemia brine shrimps twice per week, and seawater in the tanks was stagnant and exchanged 2-3 times per week. All populations were maintained under the identical baseline conditions in Percival Scientific Incubators (Model I-22LLVL, USA). Menthol-induced bleaching [49] was used to generate aposymbiotic individuals of each clonal line. Cultures of the strains SSA01 and SSB01 were used to perform inoculations with Symbiodinium and Breviolum taxa, respectively. Further, Symbiodiniaceae from the Red Sea host line were isolated (Table 1). For a detailed description of the methods refer to Supplementary Methods S1.

\section{Effect of temperature on symbiosis establishment}

Inoculations were performed using uniform mixtures containing even proportions of the strains SSA01, SSB01, and two monoclonal cultures that were grown from the previously isolated RS (Red Sea) symbionts (Table 1 and Fig. 1a). Algal strains were grown in axenic cultures for more than 1 year before conducting experiments. Cell density of each culture was assessed using a flow cytometer (BD LSRFortessa, BD Biosciences, USA) to pool equal proportions of each symbiont in the final mixture. In a flow hood, each culture flask was mixed well, and an aliquot was transferred to a $50 \mathrm{~mL}$ Falcon tube. The symbiont mixture was centrifuged for $5 \mathrm{~min}$ at $3000 \times g$, f/ 2 media was removed, and cells were re-suspended in $30 \mathrm{~mL}$ of autoclaved seawater. The cell suspension was then poured into each $250 \mathrm{~mL}$ tank containing the aposymbiotic anemones, followed by feeding with freshly hatched Artemia to facilitate the dinoflagellate uptake. Immediately after, half of the tanks were transferred to $32^{\circ} \mathrm{C}$ and the others remained at $25^{\circ} \mathrm{C}$ (Fig. 1b). The inoculated batches were left overnight, a experimental subjects

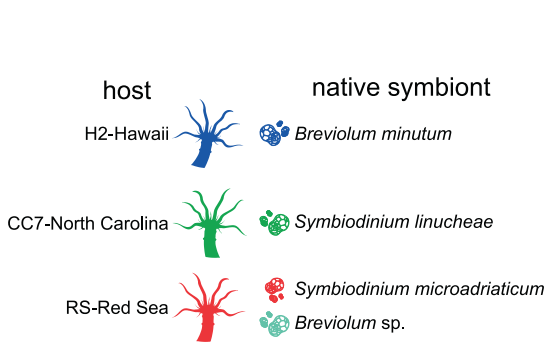

C after 12 weeks inoculation period the effect of temperature on colonization to assess

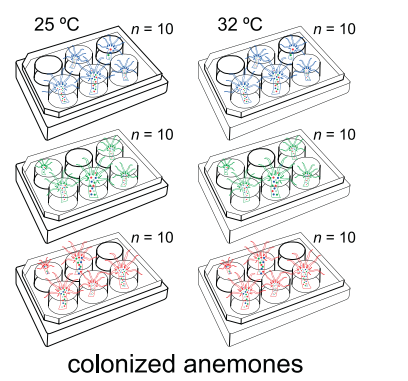

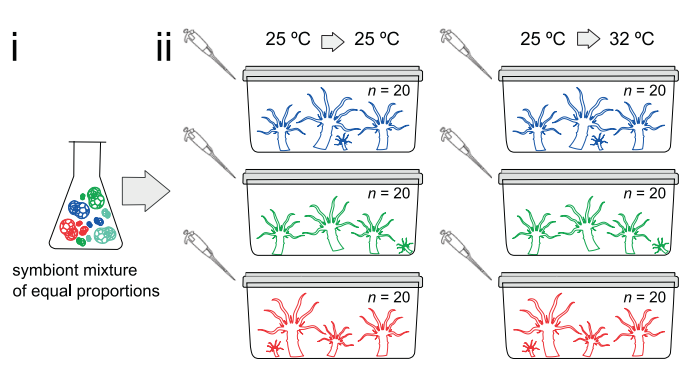

bleached anemones

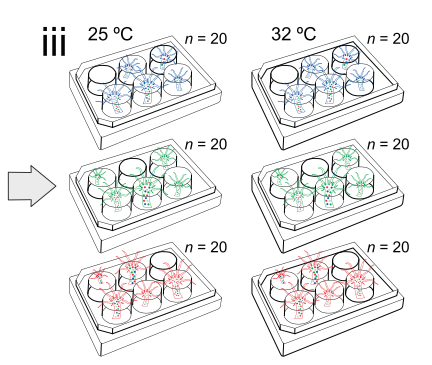

after 1 week

d elevated tempreture experiment (all anemones were subjected to $32^{\circ} \mathrm{C}$ )

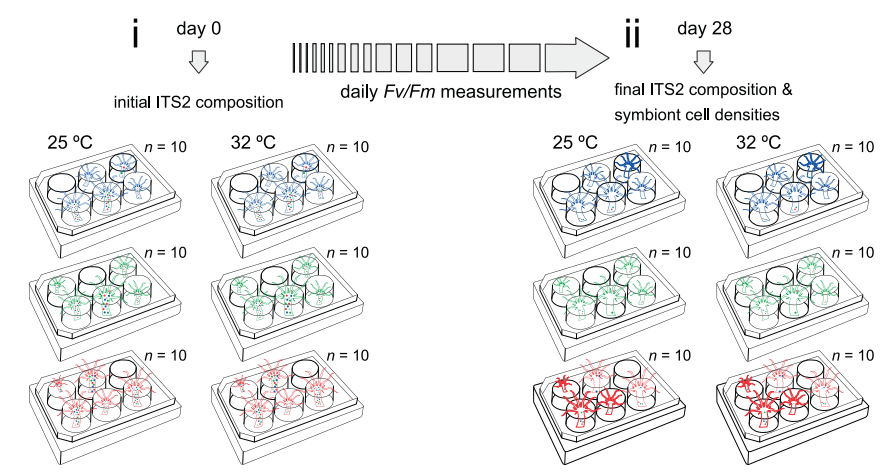

signs of bleaching

Fig. 1 Schematic diagram of the experimental design of this study. a Aiptasia host and symbiont strains used to perform inoculations. b (i) A symbiont mixture of equal proportions of all four taxa was used to inoculate (long-term acclimated to $25^{\circ} \mathrm{C}$ ) aposymbiotic anemones from of all three host lineages. (ii) Half of all individuals $(n=20$ of each genotype) were kept at $25^{\circ} \mathrm{C}$ and the rest were transferred to $32{ }^{\circ} \mathrm{C}$. (iii) Polyps were placed in six-well plates (one anemone per well, five anemones per plate). c After a 12 weeks inoculation period, symbiont cell densities were assessed for few individuals $(n=10)$ each treatment. d The remaining individuals $(n=10$ per host per temperature) were subjected to $32{ }^{\circ} \mathrm{C}$ for 28 consecutive days (25 and $32^{\circ} \mathrm{C}$ labels indicate the temperature at which individuals were inoculated). (i) Initial (day 0) and (ii) final (day 28) ITS2 composition were examined, as well as symbiont cell densities. 
and seawater was exchanged the next day. Inoculations for each treatment ( 3 hosts $\times 2$ temperatures) were performed separately so each tank (six in total) had at least 20 anemones. The above procedure was repeated three more times over the course of a week, after which polyps were transferred to six-well plates (one anemone per well) (Fig. 1b). In this way, inoculations (of the three hosts) served as independent batches, while the subsequent maintenance of anemones in individual wells provided independent biological replicates.

Inoculations were verified by fluorescence microscopy (Leica DM3000 B inverted phase contrast microscope, Leica Microsystems GmbH, Germany) every day for the first 2 weeks; until color pigmentation was visible to the naked eye. After a 12-week period, when colonization rates were generally stable [49], at least ten polyps from each condition were sacrificed to assess the effect of temperature on colonization success (measured by symbiont cell densities) (Fig. 1c), The remaining individuals (ten anemones of each group) were used in the elevated temperature experiment (Fig. 1d). Noteworthy, some Aiptasia were already acclimated to $32{ }^{\circ} \mathrm{C}$ (i.e., 12 weeks inoculation period), thus only anemones that were originally at $25^{\circ} \mathrm{C}$ and then transferred to $32{ }^{\circ} \mathrm{C}$ can be considered as heat stressed. A tentacle from each individual was taken for DNA extraction to examine the Internal Transcriber Space 2 (ITS2) compositions (at day 0) before subjecting it to $32{ }^{\circ} \mathrm{C}$ for 28 consecutive days, during which daily maximum photochemical efficiencies $(F v / F m)$ (here used as a proxy of thermal response) were recorded with a Pulse Amplitude Modulated fluorometer (Mini-PAM, Walz, Germany). For this, anemones were incubated in darkness for 30 min before taking $F v / F m$ measurements. After being under elevated temperature for 28 day, tentacles were sampled to assess the ITS2 compositions again, and individuals were analyzed for symbiont counts and protein content (Fig. 1d). In all cases where anemones from $25^{\circ} \mathrm{C}$ were transferred to $32{ }^{\circ} \mathrm{C}$, individuals were acclimated over the course of $8 \mathrm{~h}$ at increments of $1{ }^{\circ} \mathrm{C}$ per $\mathrm{h}$ (as previously described in [46]). Five days prior to exposure to elevated temperature, feeding was interrupted but later resumed after day 11 .

\section{Sample processing and ITS2 sequencing}

Details on DNA extractions, protein content analysis, and symbiont counts are provided in Supplementary Methods S2. Amplification and sequencing of the ITS2 region was done using the primers (Illumina adapters underlined below) SYM_VAR_5.82S2 (5'TCGTCGGCAGCGTCAG ATGTGTATAAGAGACAG-GAATTGCAGAACTCCGT GAACC $3^{\prime}$ ) and SYM_VAR_REV (5'GTCTCGTGGG CTCGGAGATGTGTATAAGAGACAG-CGGGTTCWCT TG TYTGACTTCATGC $3^{\prime}$ ) [50]. Library preparation is described in detailed in Supplementary Methods S3.

\section{Identification of Symbiodiniaceae taxa}

Sequencing data were analyzed using the SymPortal analytical framework [51]. SymPortal was run locally and all samples, including samples from the isolate symbiont cultures used to perform the inoculations, were analyzed together. Briefly, this software identifies sets of specific ITS2 sequences that re-occur in sufficient numbers of samples and considers them as "defining intra-genomic variants" (DIVs), which are then used to predict an "ITS2 type profile" representative of putative Symbiodiniaceae taxa. However, due to the nature of our data and the availability of pure culture sequence profiles, we used the pre-med file from SymPortal, which represents the sequence associations before the minimum entropy decomposition analyses (i.e., step in which SymPortal consolidates sequences into a smaller set). This approach allowed us to identify the diagnostic (i.e., distinct and most abundant) sequence of each symbiont strain and thus resolve mixed samples in a more accurate manner (see Supplementary Methods S4 and Figs. S1 and S2). Based on this, we identified three main putative taxa: B1, A4, and A1, corresponding to B. minutum from Hawaii (SSB01), S. linucheae (SSA01), and S. microadriaticum (RS-A), respectively. The minor sequence variant 1369 B, on the other hand, was diagnostic of SSB01 as it was not present in the pure Red Sea culture, which we designated RS-B1. The ratio of the minor variant 1369_B to the major B1 sequence thus allowed us to quantitatively resolve the relative abundances of SSB01 and RS-B1 in mixes (see additional details in Supplementary Methods S4 and Table S1). Sequences belonging to other taxa with abundances below $1 \%$ were classified as "others".

\section{Data analyses}

\section{Part I: Differences in Symbiodiniaceae communities}

All data analyses were conducted in $\mathrm{R}$ version 3.5.1 [52] using the package "vegan" [53] to test for differences in the interaction, abundance, and composition of Symbiodiniaceae communities among the fixed and orthogonal explanatory variables; host, temperature, and time point (day). Prior to any analysis, count data (DIVs) were $\ln (x+1)$ transformed. Homogeneity of multivariate dispersion (based on BrayCurtis distances) for each factor was tested with permutation tests of multivariate dispersion (PERMDISP [54]) using the function "betadisper" and set to 9999 permutations. Because the dispersion of samples was significantly different between groups (Table S2), multivariate statistical analyses were conducted per sample type (i.e., separately for H2-Hawaii, CC7-North Carolina, and RS-Red Sea). Two-factorial permutational multivariate analysis of variance [55] were run 
with 9999 permutations to test for effects of temperature and time in a full-factorial design using the function "adonis". Differences between treatments were then visualized with principal coordinate analyses plots and further tested using canonical analysis of principal coordinates (CAP) with 9999 permutations [56] using the function "CAPdiscrim" from the "BiodiversityR" package. Finally, negative binomial distribution models (as implemented in DESeq2 [57]) were used to detect differentially abundant DIVs as described previously in [25]. Comparisons included differential abundance testing among relevant temperature and time interactions (Fig. S3). In all cases, $p$ values were adjusted with the BenjaminiHochberg correction method.

\section{Part II: Response variables measured}

The biological response variables were analyzed using both ANOVAs and repeated linear mixed models (LMMs). All data were first checked for normality and homoscedasticity using standardized residual plots and Q-Q plots and, if required, data were $\ln (x+1)$ transformed. Maximum photochemical efficiencies were analyzed using repeatedmeasures LMMs in SPSS [58]. The fixed factors were host genotype, pre-exposure temperature and time, which was the repeated measure. Due to the unavoidably low and uneven replication among hosts (Fig. 2), symbiont composition was not included as a factor in the analysis. Further, preliminary analyses revealed differences in $F v / F m$ trends among hosts regardless of their symbiont composition (either at day 0 or day 28). Given that the presence of feeding co-occurred with the factor of time, feeding was not included as a factor, but data were interpreted accordingly. Several repeated covariance types [e.g., $\mathrm{AR}(1), \mathrm{AR}(1)$ heterogeneous, CS] were investigated to assess the model of best fit by comparing various goodness-of-fit statistics [e.g., -2 restricted log likelihood, Akaike's information criterion, and Bayesian information criterion]. Estimated marginal means (least-squares means) were used to determine which means differed for the significant, highest-order terms. Finally, symbiont cell densities were analyzed with a two-way ANOVA in R using host and temperature as fixed factors. Tukey pairwise comparisons were further conducted post hoc to determine where significant differences occurred.

\section{Results}

\section{Temperature effect on symbiont acquisition and maintenance}

Overall, Aiptasia from $25^{\circ} \mathrm{C}$ (regardless of the host genotype) were initially (day 0 ) dominated by Breviolum taxa compared to those from $32^{\circ} \mathrm{C}$, which had much higher abundances of A1 (Fig. 2). Symbiodiniaceae communities
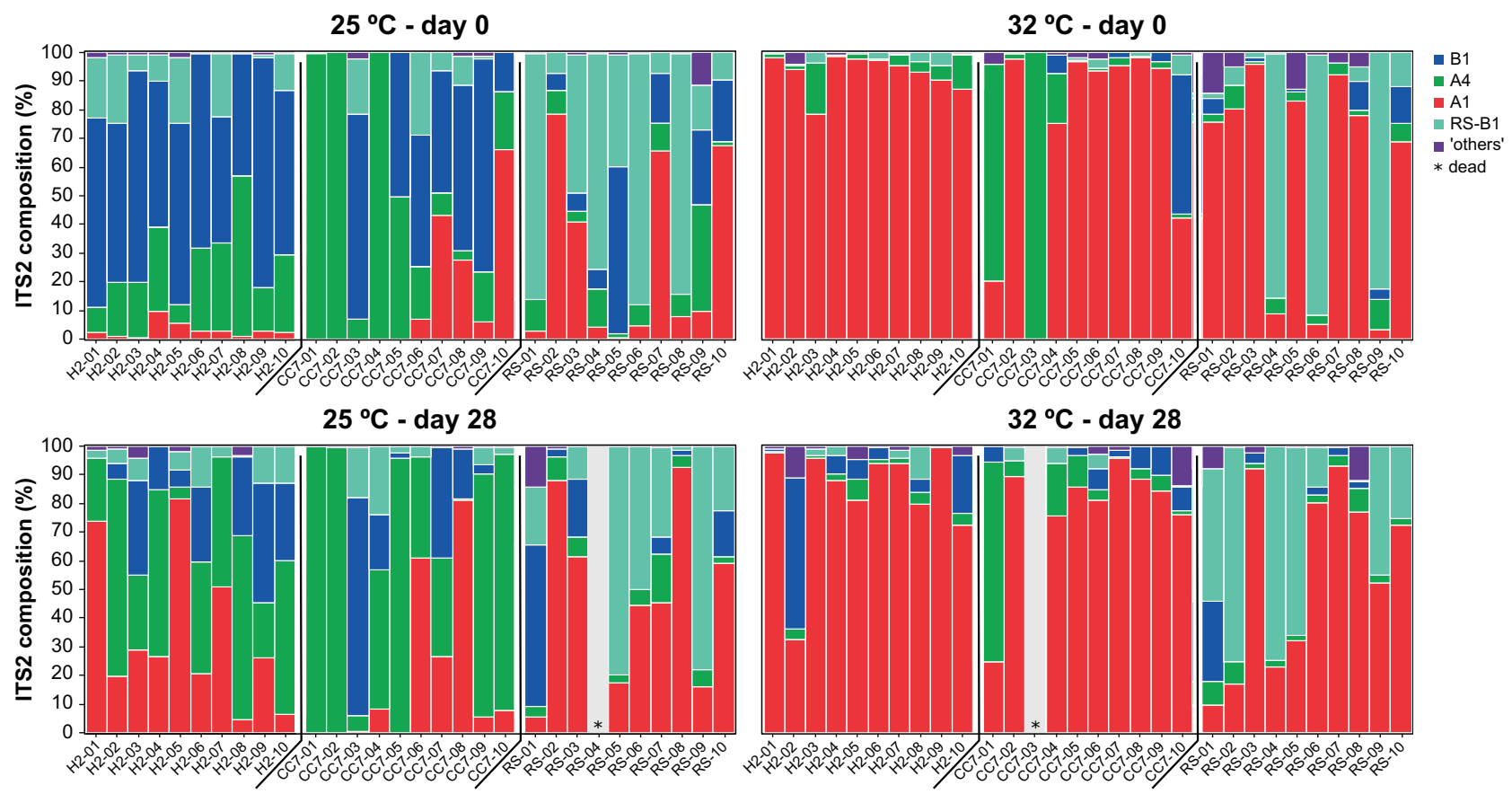

Fig. 2 ITS2 composition of the Symbiodiniaceae communities of different Aiptasia colonized at 25 and $32{ }^{\circ} \mathrm{C}$ before (day 0 ) and after (day 28) being subjected to elevated temperature $\left(32^{\circ} \mathrm{C}\right)$. Putative taxa are reported based on their diagnostic sequences and its taxonomic description as referenced in the literature: B1 (B. minutum

and B. antillogorgium and B. pseudominutum), A4 (S. linucheae), and A1 (S. microadriaticum). B1 taxa from the Red Sea isolate are designated as RS-B1. Sequences belonging to other taxa were classified as "others". 
across all treatments were well segregated (explaining up to $87 \%$ of the total variability, Fig. 3a), mainly according to temperature (Fig. 3b). Indeed, significant differences were detected for all groups (Table S3) when analyzed separately (see Fig. 3c, d for H2-Hawaii, Fig. 3f, g for CC7-North Carolina, and Fig. 3i, j for RS-Red Sea). Symbiont composition appeared to differ across time; in particular, anemones from $25^{\circ} \mathrm{C}$ showed a substantial increase (up to threefold) of A1 sequences after being exposed to elevated temperature for 28 days (Fig. $3 \mathrm{e}, \mathrm{h}, \mathrm{k}$ ). Furthermore, analyses of raw abundances revealed a high number of differentially abundant DIVs across treatments (Table S4). Notably, we detected DIVs associated with Symbiodinium (clade A), Cladocopium (clade C), and/or Durusdinium (clade D). Abundances of these types were significantly higher in communities from $32^{\circ} \mathrm{C}$, particularly at day 0 but also after transferring the $25^{\circ} \mathrm{C}$ individuals to this treatment (Fig. S4). Conversely, Breviolum (clade B) variants were generally underrepresented in the $32{ }^{\circ} \mathrm{C}$ treatment, despite these being the most abundant taxa across all groups. Finally, elevated temperature also had an overall effect on symbiont colonization as evidenced by the lower cell densities in anemones from $32{ }^{\circ} \mathrm{C}$ (though this was significantly different for $\mathrm{CC} 7$-North Carolina only, Fig. 4).

\section{Extent of thermal response depends on host genotype and pre-exposure treatment}

We observed a rapid decline in photochemical efficiencies $(F v / F m)$ from $\sim 0.9$ to $\sim 0.7$ during the first 10 days of exposure. However, after feeding resumed at day $11, \mathrm{Fv} / \mathrm{Fm}$ values increased and steadily returned to their initial values by the end of the exposure (day 28) (Fig. 5a, b). Contrary to our expectations, both pre-exposure treatments exhibited similar response patterns (Fig. 5a, b), but the response over time depended on host type, resulting in a significant host $x$ day interaction $(p<0.001)$. Specifically, the same ranking of host responses (RS-Red Sea $>$ CC7-North Carolina $>$ H2Hawaii) was observed for both treatments $(p<0.05)$, whereby RS-Red Sea consistently exhibited the highest $F v$ / Fm values and H2-Hawaii the lowest. Importantly, however, our analysis detected a significant host $\times$ pre-exposure treatment $(p<0.001)$ interaction (Table 2), which resulted from differences between $F v / F m$ responses of the preexposure treatments regardless of time and the inconsistency of this pattern across host types (Fig. 5c). Indeed, within host comparisons revealed an effect of thermal preexposure in the response to elevated temperature (Fig. 5c), but this effect was primarily driven by higher photochemical efficiency in the H2-Hawaii individuals from $32{ }^{\circ} \mathrm{C}$ compared to their counterparts from $25^{\circ} \mathrm{C}(p<0.001$, Fig. 5c). These data are highly consistent with the significantly greater density of symbionts in pre-exposed hosts after 28 days at $32{ }^{\circ} \mathrm{C}$ (Fig. 6).

\section{Discussion}

\section{Temperature predicts attributes of Symbiodiniaceae communities across treatments}

Here, we studied the dynamics of multi-species symbioses establishment in Aiptasia hosts from different regions under "optimal" and elevated temperature conditions using only symbionts that are native to our model system. In accordance with [12, 32], our data are consistent with that symbionts' thermotolerance followed the order of $S$. linucheae (A4) $\geq S$. microadriaticum (A1) $>$ Breviolum from the Red Sea (RS-B1) $>B$. minutum from Hawaii (B1). Considering previous evidence for symbiont shuffling and/ or switching $[17,18]$, we hypothesized that communities would shift from Breviolum-dominated toward more heat tolerant taxa as temperature increased. Indeed, we observed a significantly higher abundance of variants associated with Symbiodinium (clade A), Cladocopium (clade C), and Durusdinium (clade D) taxa in anemones that were preexposed to $32{ }^{\circ} \mathrm{C}$, but also in heat stressed individuals (that is, Aiptasia reared at $25^{\circ} \mathrm{C}$ and subsequently subjected to $32^{\circ} \mathrm{C}$ ).

Symbiodinium species are known to be more resilient, with lower reactive oxygen species levels and slower deterioration of photosystem (PS) II compared to Breviolum, for example [41, 59-62]. Surprisingly, despite $S$. linucheae (A4) being supposedly the most thermally tolerant species tested here (according to [32]), it was barely observed at $32{ }^{\circ} \mathrm{C}$; only in few individuals from $25^{\circ} \mathrm{C}$ and for which it was already present in their initial ITS2 composition. Instead, high abundances of $S$. microadriaticum (A1) were detected. We can only presume that in these particular settings, A1 symbionts were more beneficial and/ or competitive than $\mathrm{A} 4$, at least in the short term. In addition, we expected a shift from B1 to RS-B1 for individuals that were transferred from 25 to $32{ }^{\circ} \mathrm{C}$, however, this was not observed. Moreover, besides having a significant effect in community composition, temperature also moderated the host's uptake of symbionts. Particularly, and consistent with previous observations [29], lower cell densities (though only significantly different for CC7-North Carolina) were attained in inoculations from $32^{\circ} \mathrm{C}$.

Gabay et al. found strong limitations in the acquisition of thermally tolerant symbionts due to the high specificity of H2-Hawaii Aiptasia (which they refer to as a population from the Indo-Pacific that associates mainly with $B$. minutum). However, considering that they used nonnative, foreign symbionts, it is unsurprising that these 

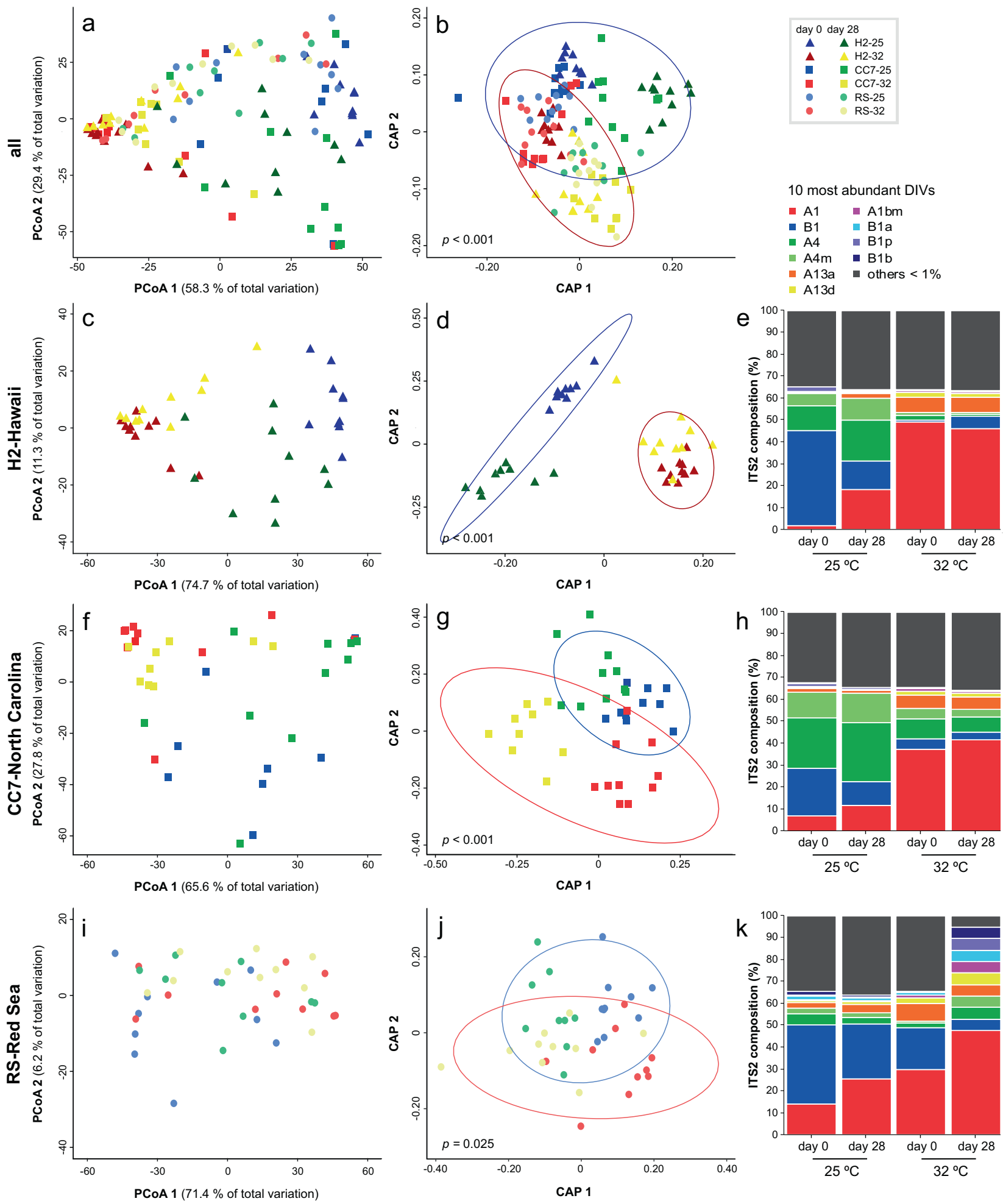

Fig. 3 Distinct Symbiodiniaceae communities associated with different Aiptasia hosts at two temperatures after 12 weeks of performing inoculations. Principal coordinate analysis (PCoA) showing variation in the ITS2 composition of the Symbiodiniaceae communities associated with (a) all Aiptasia from 25 and $32{ }^{\circ} \mathrm{C}$ before (day 0 ) and after (day 28) being subjected to elevated temperature and for $(\mathbf{c}, \mathbf{f}, \mathbf{i})$ each host separately. Constrained analysis of the canonical axes of the principal coordinates (CAP) show significant differences $(p<0.001)$ between temperature and time treatments $(\mathbf{b}, \mathbf{d}, \mathbf{g}, \mathbf{j})$. Different shades of blue and green represent treatments at $25^{\circ} \mathrm{C}$ (day 0 and 28 , respectively), whilst red and yellow colors show communities from $32{ }^{\circ} \mathrm{C}$ (likewise). Ellipses represent the $95 \%$ confidence intervals for the temperature factor (blue for $25^{\circ} \mathrm{C}$ and red for $32^{\circ} \mathrm{C}$ ). Taxonomy bar charts show the relative abundance of the most abundant taxa for each group $(\mathbf{e}, \mathbf{h}, \mathbf{k})$. All DIVs for which abundance was below $1 \%$ were grouped together in the "others" category. 
failed to establish symbiosis $[24,42,63,64]$. Contrary to the above, we show that (elevated) temperature voids inter-partner fidelity and instead promotes associations with different Symbiodiniaceae. Indeed, various symbiont strains can colonize Aiptasia under laboratory conditions [23, 24, 63, 64], yet, natural populations are known to engage in highly specific symbioses, such that genetic differences in the anemone host correlate with genetic differences in the algal symbiont [65]. Thus, we do not know to what extent environment overrules symbiosis specificity. For instance, despite the strong effect of temperature on symbiont composition, we still observed strain preference to a certain degree, given that H2-Hawaii and RS-Red Sea anemones had more B1 and RS-B1, respectively, at least at $25^{\circ} \mathrm{C}$.

Neither [33] nor our study investigated colonization dynamics beyond a few weeks (4 months in our case),

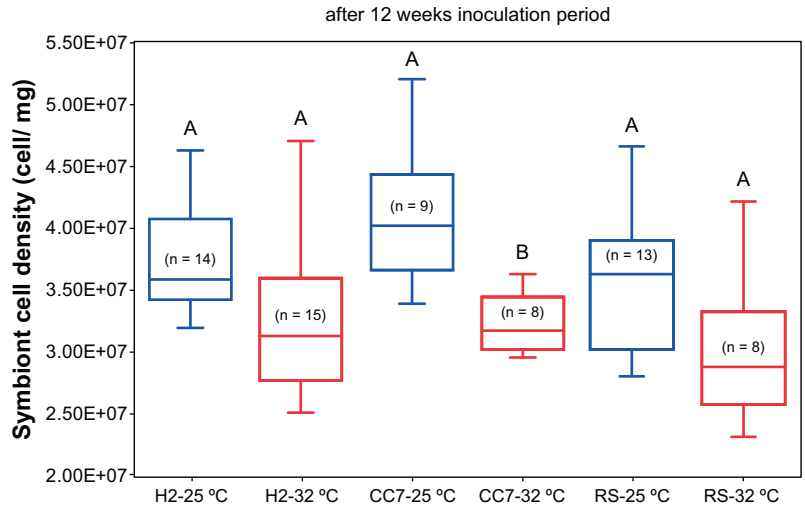

Fig. 4 Mean $( \pm 1 \mathrm{SE})$ symbiont cell densities (normalized to protein content) of different Aiptasia from $25^{\circ} \mathrm{C}$ (blue) to $32^{\circ} \mathrm{C}$ (red) after 12 weeks of performing inoculations. Pairwise comparisons were carried out within each host genotype. Letters above error bars indicate similarities (e.g., AA) or differences (e.g., AB) between host-symbiont combinations, as determined by Tukey's HSD post hoc test $(p<0.05)$. which is extremely important as symbioses are not stable and can change with time. Whether heat tolerant symbionts can be retained over the lifetime of corals remains an open question. More resilient taxa usually come at high energetic costs and reduced growth [22, 66, 67]. Indeed, most adult colonies harbor only one predominant species/genotype, which would be the more beneficial for the host [68], and not necessarily the most thermal tolerant. Predicting the fate of novel partnerships, especially under stress conditions, is not simple. We can only hypothesize that these trade-offs will decrease as temperatures increase, thus promoting the establishment and proliferation of heat resistant symbioses [26].

\section{Pre-exposure to elevated temperature improves the thermal response of the Aiptasia holobiont}

Numerous studies (reviewed in [44]) have demonstrated that the thermal resilience of corals can be improved by rearing juvenile (developmental acclimation) and adult stages (acclimation) at higher temperatures for days or weeks, and/ or exposing them to sub-lethal levels of heat stress (hardening) for even just a few hours. Our findings concur with these observations, that is, thermal response (which here we assessed by measuring the PSII operating efficiency) of holobionts preconditioned to elevated temperature improved compared to that of individuals from $25^{\circ} \mathrm{C}$. Specifically, $\mathrm{Fv} / \mathrm{Fm}$ of anemones that were inoculated at $32{ }^{\circ} \mathrm{C}$ seemed to be higher than those from $25^{\circ} \mathrm{C}$. However, we also showed that any fitness advantage conferred by temperature-induced acclimation was severely affected by food deprivation, which suggests that the processes involved are energy demanding. Indeed, factors like sex, age, ontogeny, and food availability are known to affect the thermal responses of a wide range of ectotherms [69-71], in addition to inter-individual and species variation. a

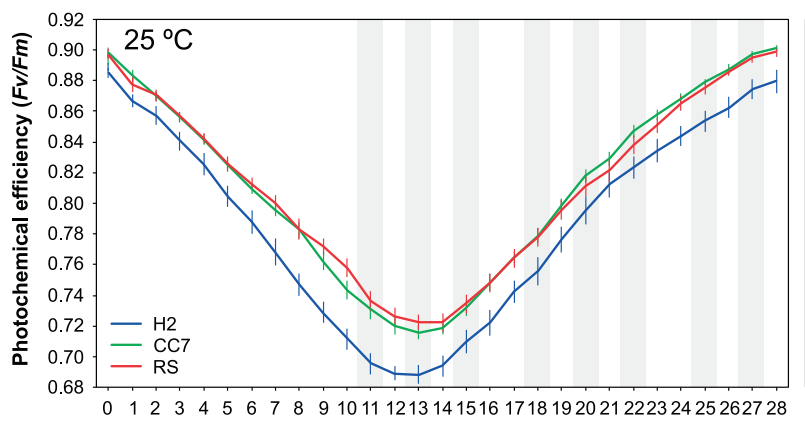

Fig. 5 Photophysiological response of different Aiptasia holobionts from two temperatures is affected by food availability. Mean $( \pm 1 \mathrm{SE})$ photochemical efficiencies of different Aiptasia pre-exposed to (a) $25^{\circ} \mathrm{C}$ and (b) $32{ }^{\circ} \mathrm{C}$ before (day 0) and throughout 28 days of being subjected to elevated temperature. $\mathbf{c}$ Overall differences in PSII b

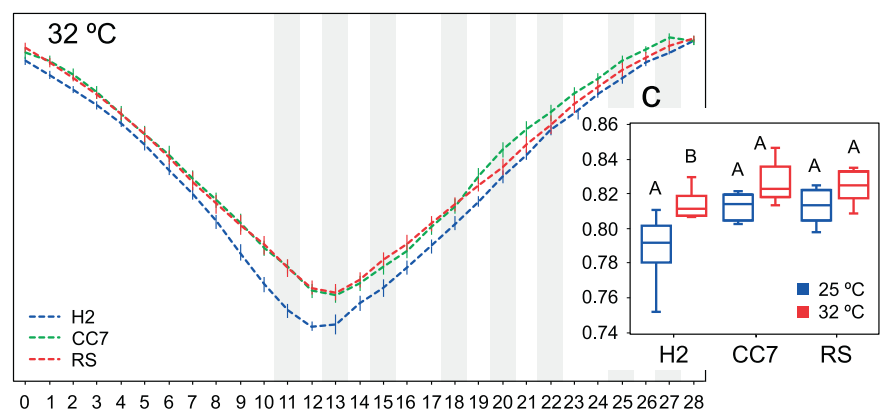

operating efficiency across groups. Shaded areas indicate days in which animals were fed. Letters indicate overall similarities (e.g., AA) or differences (e.g., $\mathrm{AB}$ ), as determined by estimated marginal means, between holobionts from different temperatures $\left(25^{\circ} \mathrm{C}\right.$ vs $\left.32^{\circ} \mathrm{C}\right)$. 
Table 2 Summary of results of a linear mixed-model (LMM) analysis comparing photochemical efficiencies $(F v / F m)$ of different Aiptasia holobionts that were inoculated at 25 and $32{ }^{\circ} \mathrm{C}$ before (day 0 ) and after (day 28) being subjected to elevated temperature.

\begin{tabular}{|c|c|c|c|c|}
\hline $\begin{array}{l}\text { Source of } \\
\text { variation }\end{array}$ & Numerator df & Denominator df & $F$ & $p$ \\
\hline Host & 2 & 89.742 & 33.404 & $<0.001$ \\
\hline $\begin{array}{l}\text { Pre- } \\
\text { exposure temp }\end{array}$ & 1 & 89.742 & 8.381 & 0.041 \\
\hline Day & 28 & 1146.153 & 266.155 & $<0.001$ \\
\hline $\begin{array}{l}\text { Host } \times \text { pre- } \\
\text { exposure temp }\end{array}$ & 2 & 89.742 & 10.900 & $<0.001$ \\
\hline Host $\times$ day & 56 & 1146.153 & 1.871 & $<0.001$ \\
\hline $\begin{array}{l}\text { Pre-exposure } \\
\text { temp } \times \text { day }\end{array}$ & 28 & 1146.153 & 0.886 & 0.637 \\
\hline $\begin{array}{l}\text { Host } \times \text { pre- } \\
\text { exposure } \\
\text { temp } \times \text { day }\end{array}$ & 56 & 1146.153 & 1.089 & 0.308 \\
\hline
\end{tabular}

Data were $\operatorname{Ln}(x+1)$ transformed. The model of best fit was AR(1). AIC (Akaike information criterion) $=-11,760.310$, BIC (Bayesian information criterion) $=-11,749.712$. Statistically significant $p$ values (0.05) are denoted in italics. In each case, the significant sources of variation are shown in bold.

$d f$ degrees of freedom.

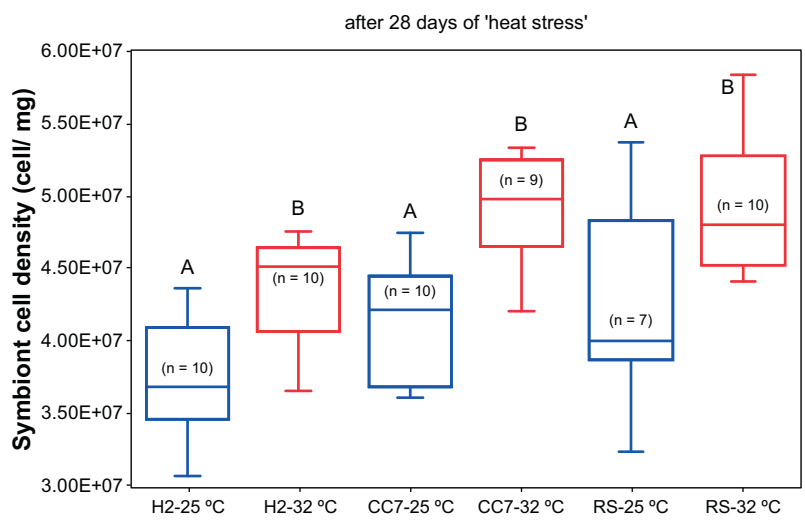

Fig. 6 Mean $( \pm 1 \mathrm{SE})$ symbiont cell densities (normalized to protein content) of different Aiptasia from $25^{\circ} \mathrm{C}$ (blue) to $32{ }^{\circ} \mathrm{C}$ (red) after being subjected to 28 days of elevated temperature. Pairwise comparisons were carried out within each host genotype. Letters above error bars indicate similarities (e.g., AA) or differences (e.g., AB) between host-symbiont combinations, as determined by Tukey's HSD post hoc test $(p<0.05)$

Elevated temperatures affect both the host and photosymbiont, yet, it has long been suggested that the latter ultimately determines corals' resilience to stress [8, 9, 11]. Recent work [72] has shown, however, that the host environment (e.g., variations in metabolism) can strongly affect the physiology of even the most heat tolerant symbiont types and thus, alter the overall performance of the holobiont. Indeed, a given host provides specific growth conditions (e.g., in hospite nutrient availability) for the symbiont community which, in turn, affects the productivity of the holobiont system [73]; and so reflects on the ability to (physiologically) compensate for stress in regard to its energetic demands (i.e., environment provided for the symbiont). Our findings support this notion, indicating that thermal response of the Aiptasia holobiont might be fundamentally determined by host genotype. Particularly, CC7North Carolina and RS-Red Sea have been identified as more (genotypic- and phenotypically) plastic, thermal resistant lineages [39]. Indeed, both CC7-North Carolina and RS-Red Sea (although not different from each other) exhibited higher photochemical efficiencies and greater densities of symbionts than H2-Hawaii regardless of their pre-exposure to elevated temperature. These observations highlight the potential of using Red Sea Aiptasia to study thermal resilience of symbiotic cnidarians, as they thrive in one of the hottest (as high as $35^{\circ} \mathrm{C}$ during summer) seas on Earth and naturally experience temperatures predicted elsewhere for the end of the century under "business as usual" emissions pathways without bleaching [74, 75].

\section{Effect of active feeding during thermal stress}

While it was not our intention to specifically test for the effect of food availability, it is not uncommon to interrupt feeding before measuring physiological variables in order to reduce the effect of trophic status [46, 73, 76, 77]. In the present study, animals were unfed prior to (up to 3 days) and partially over the course of the experiment (first 10 days) and so, we cannot disentangle the confounded influence of food availability and temperature. Nevertheless, feeding certainly has a major effect on the response to temperature [78-81]; thus, it is not surprising that Aiptasia from $32{ }^{\circ} \mathrm{C}$ also showed signs of stress (as seen by the decline in $F v / F m$ ) when deprived from food. Indeed, previous studies [79, 82] have shown that the photochemical efficiency of fed corals is up to $70 \%$ higher compared to starved individuals under thermal stress (but see [83]).

\section{Methodological considerations}

Overall, our results showed that symbiont communities differed substantially among temperature treatments. However, as samples were only taken at the beginning (day 0 ) and end (day 28) of the experiment, we cannot pinpoint exactly when these changes occurred. It is possible that some individuals were not fully aposymbiotic at the time we performed the inoculations, which could have contributed to the proliferation of symbiont types that were not included in the inoculation mixture. Such was the case for Cladocopium and Durusdinium taxa that might have been initially present in very low abundances and then increased at elevated temperature (as they are more heat resistant). However, 
while we cannot exclude potential contamination through incomplete bleaching, feeding (i.e., food was taken from a common batch), and/or subsequently during PCR amplification, our conclusions remain the same; that is, temperature has a greater effect on symbiont composition than partner specificity.

Here, we attributed differences in thermal performance to host genotype and pre-exposure temperature only due to two non-mutually exclusive reasons: (1) low and uneven replication level of most host-symbiont combinations did not allow us to include the symbiont factor in our analyses (i.e., not enough power to detect differences), and (2) we do not know when symbiont communities changed, and if this was the result of elevated temperature alone or if feeding also had an effect. Thus, we are cautious when interpreting our results, as we disregarded any contribution from the symbiont partner but are also unable to disentangle the combined effects of temperature and food deprivation. Nevertheless, our data strongly indicate that symbiont identity played a critical role in moderating the thermal response of the holobionts and thus, their role should not be underestimated. To further elucidate the exact nature of the drivers at play, higher replication of the different hostsymbiont combinations across conditions and control treatments (i.e., keeping animals at $25^{\circ} \mathrm{C}$ for the 28 days period and with continuous feeding) is likely required. It should be noted that food deprivation can have a significant effect on protein content, which here we used as a reference to normalize symbiont counts. For example, studies [84, 85] have shown that fed corals (even in moderate levels, i.e., twice a week) have up to $49 \%$ higher protein content than starved corals. Thus, it is not surprising that symbiont cell/ protein ratios differ across experiments such that symbiont cell densities cannot be compared. One final consideration noteworthy of mentioning is that the salinity of the seawater we used to rear all Aiptasia ( $\sim 39$ psu from the central Red Sea) is significantly higher than other studies. The latter is relevant in the context of this study as it has recently been shown that high salinity levels can contribute to greater thermotolerance of cnidarian holobionts [46] by modulating the physiology of its photosymbionts [86].

\section{Conclusions}

Here, we used the flexible coral model Exaiptasia pallida and deep sequencing of symbiont communities to assess the establishment and performance of symbioses under different thermal conditions. Our results show that temperature has a significant and greater effect than partner specificity on the symbiont composition of Aiptasia, with increased temperature favoring colonization (and proliferation) of more heat resistant types. Our data add to the body of work showing that pre-exposure to elevated temperature is crucial for the thermal resilience of the cnidarian holobiont, which is also dependent on other factors like, in this case, food availability.

\section{Data availability}

Sequencing data are available at NCBI under BioProject ID number PRJNA577392.

Acknowledgements We thank the KAUST Bioscience Core Laboratory for library sequencing and Luke Esau for assisting with flow cytometry. We thank Dr. Ben Hume for support with the SymPortal analysis.

Funding Research reported in this publication was supported by funding from KAUST.

Author contributions MA conceived the idea and obtained funding. $\mathrm{MA}$ and $\mathrm{MH}$ designed experiments. $\mathrm{MH}, \mathrm{SC}$, and JEC performed experiments in the laboratory. MH, MA and SGK analyzed and interpreted data. AP assisted with bioinformatic analyses. MH, MA, SK and CD wrote the first draft of the manuscript with input from all authors. All authors read and approved the final manuscript.

\section{Compliance with ethical standards}

Conflict of interest The authors declare that they have no conflict of interest.

Publisher's note Springer Nature remains neutral with regard to jurisdictional claims in published maps and institutional affiliations.

Open Access This article is licensed under a Creative Commons Attribution 4.0 International License, which permits use, sharing, adaptation, distribution and reproduction in any medium or format, as long as you give appropriate credit to the original author(s) and the source, provide a link to the Creative Commons license, and indicate if changes were made. The images or other third party material in this article are included in the article's Creative Commons license, unless indicated otherwise in a credit line to the material. If material is not included in the article's Creative Commons license and your intended use is not permitted by statutory regulation or exceeds the permitted use, you will need to obtain permission directly from the copyright holder. To view a copy of this license, visit http://creativecommons. org/licenses/by/4.0/.

\section{References}

1. Hoegh-Guldberg O. Climate change, coral bleaching and the future of the world's coral reefs. Mar Freshw Res. 1999;50:839-66.

2. Hoegh-Guldberg O, Poloczanska ES, Skirving W, Dove S. Coral reef ecosystems under climate change and ocean acidification. Front Mar Sci. 2017;4:158.

3. Kenkel CD, Goodbody-Gringley G, Caillaud D, Davies SW, Bartels E, Matz MV. Evidence for a host role in thermotolerance divergence between populations of the mustard hill coral (Porites astreoides) from different reef environments. Mol Ecol. 2013;22: 4335-48.

4. Bay RA, Palumbi SR. Multilocus adaptation associated with heat resistance in reef-building corals. Curr Biol. 2014;24:2952-6. 
5. Dixon GB, Davies SW, Aglyamova GV, Meyer E, Bay LK, Matz MV. Genomic determinants of coral heat tolerance across latitudes. Science. 2015;348:1460.

6. Howells EJ, Abrego D, Meyer E, Kirk NL, Burt JA. Host adaptation and unexpected symbiont partners enable reef-building corals to tolerate extreme temperatures. Glob Change Biol. 2016;22:2702-14.

7. Morikawa MK, Palumbi SR. Using naturally occurring climate resilient corals to construct bleaching-resistant nurseries. Proc Natl Acad Sci USA. 2019;116:10586.

8. Berkelmans R, van Oppen MJH. The role of zooxanthellae in the thermal tolerance of corals: a 'nugget of hope' for coral reefs in an era of climate change. Proc Biol Sci. 2006;273:2305-12.

9. Sampayo EM, Ridgway T, Bongaerts P, Hoegh-Guldberg O. Bleaching susceptibility and mortality of corals are determined by fine-scale differences in symbiont type. Proc Natl Acad Sci USA. 2008; 105:10444.

10. LaJeunesse TC, Smith RT, Finney J, Oxenford H. Outbreak and persistence of opportunistic symbiotic dinoflagellates during the 2005 Caribbean mass coral 'bleaching' event. Proc R Soc B: Biol Sci. 2009;276:4139-48.

11. Howells EJ, Beltran VH, Larsen NW, Bay LK, Willis BL, van Oppen MJH. Coral thermal tolerance shaped by local adaptation of photosymbionts. Nat Clim Change. 2011;2:116.

12. LaJeunesse TC, Parkinson JE, Gabrielson PW, Jeong HJ, Reimer JD, Voolstra CR, et al. Systematic revision of Symbiodiniaceae highlights the antiquity and diversity of coral endosymbionts. Curr Biol. 2018;28:2570-.e6.

13. Bellantuono AJ, Granados-Cifuentes C, Miller DJ, Hoegh-Guldberg $\mathrm{O}$, Rodriguez-Lanetty M. Coral thermal tolerance: tuning gene expression to resist thermal stress. PLoS ONE. 2012;7:e50685.

14. Palumbi SR, Barshis DJ, Traylor-Knowles N, Bay RA. Mechanisms of reef coral resistance to future climate change. Science. 2014;344:895.

15. Sawall Y, Al-Sofyani A, Hohn S, Banguera-Hinestroza E, Voolstra CR, Wahl M. Extensive phenotypic plasticity of a Red Sea coral over a strong latitudinal temperature gradient suggests limited acclimatization potential to warming. Sci Rep. 2015;5:8940.

16. Torda G, Donelson JM, Aranda M, Barshis DJ, Bay L, Berumen ML, et al. Rapid adaptive responses to climate change in corals. Nat Clim Change. 2017;7:627.

17. Baker AC. Flexibility and specificity in coral-algal symbiosis: diversity, ecology, and biogeography of Symbiodinium. Annu Rev Ecol, Evolution, Syst. 2003;34:661-89.

18. Boulotte NM, Dalton SJ, Carroll AG, Harrison PL, Putnam HM, Peplow LM, et al. Exploring the Symbiodinium rare biosphere provides evidence for symbiont switching in reef-building corals. ISME J. 2016;10:2693-701.

19. Cunning R, Silverstein RN, Baker AC. Symbiont shuffling linked to differential photochemical dynamics of Symbiodinium in three Caribbean reef corals. Coral Reefs. 2018;37:145-52.

20. Stat M, Loh WKW, LaJeunesse TC, Hoegh-Guldberg O, Carter DA. Stability of coral-endosymbiont associations during and after a thermal stress event in the southern Great Barrier Reef. Coral Reefs. 2009;28:709-13.

21. Putnam HM, Stat M, Pochon X, Gates RG. Endosymbiotic flexibility associates with environmental sensitivity in scleractinian corals. Proc R Soc B: Biol Sci. 2012;279:4352-61.

22. Stat M, Morris E, Gates RD. Functional diversity in coral-dinoflagellate symbiosis. Proc Natl Acad Sci USA. 2008; 105:9256.

23. Starzak DE, Quinnell RG, Nitschke MR, Davy SK. The influence of symbiont type on photosynthetic carbon flux in a model cnidarian-dinoflagellate symbiosis. Mar Biol. 2014;161:711-24.

24. Gabay Y, Weis VM, Davy SK. Symbiont identity influences patterns of symbiosis establishment, host growth, and asexual reproduction in a model cnidarian-dinoflagellate symbiosis. Biol Bull. 2018;234:1-10.

25. Quigley KM, Bay LK, Willis BL. Temperature and water qualityrelated patterns in sediment-associated Symbiodinium communities impact symbiont uptake and fitness of juveniles in the genus acropora. Front Mar Sci. 2017;4:401.

26. Cumbo VR, vanOppen MJH, Baird AH. Temperature and Symbiodinium physiology affect the establishment and development of symbiosis in corals. Mar Ecol Prog Ser. 2018;587:117-27.

27. Ali A, Kriefall NG, Emery LE, Kenkel CD, Matz MV, Davies SW. Recruit symbiosis establishment and Symbiodiniaceae composition influenced by adult corals and reef sediment. Coral Reefs. 2019;38:405-15.

28. McIlroy SE, Cunning R, Baker AC, Coffroth MA. Competition and succession among coral endosymbionts. Ecol Evolution. 2019;9:12767-78.

29. Abrego D, Willis BL, van Oppen MJH. Impact of light and temperature on the uptake of algal symbionts by coral juveniles. PLoS ONE. 2012;7:e50311.

30. Schnitzler CE, Hollingsworth LL, Krupp DA, Weis VM. Elevated temperature impairs onset of symbiosis and reduces survivorship in larvae of the Hawaiian coral, Fungia scutaria. Mar Biol. 2012; 159:633-42.

31. Hawkins TD, Hagemeyer JCG, Warner ME. Temperature moderates the infectiousness of two conspecific Symbiodinium strains isolated from the same host population. Environ Microbiol. 2016; 18:5204-17.

32. Swain TD, Chandler J, Backman V, Marcelino L. Consensus thermotolerance ranking for 110 Symbiodinium phylotypes: an exemplar utilization of a novel iterative partial-rank aggregation tool with broad application potential. Funct Ecol. 2017;31:172-83.

33. Gabay Y, Parkinson JE, Wilkinson SP, Weis VM, Davy SK. Inter-partner specificity limits the acquisition of thermotolerant symbionts in a model cnidarian-dinoflagellate symbiosis. ISME J. 2019;13:2489-99.

34. Poland DM, Coffroth MA. Trans-generational specificity within a cnidarian-algal symbiosis. Coral Reefs. 2017;36:119-29.

35. Fraune S, Bosch TCG. Long-term maintenance of species-specific bacterial microbiota in the basal metazoan Hydra. Proc Natl Acad Sci USA. 2007;104:13146.

36. Herrera M, Ziegler M, Voolstra CR, Aranda M. Laboratorycultured strains of the sea anemone exaiptasia reveal distinct bacterial communities. Front Mar Sci. 2017;4:115.

37. Grajales A, Rodríguez E. Morphological revision of the genus Aiptasia and the family Aiptasiidae (Cnidaria, Actinaria, Metridioidea). Zootaxa. 2014;3826:55-100.

38. Xiang T, Hambleton EA, DeNofrio JC, Pringle JR, Grossman AR. Isolation of clonal axenic strains of the symbiotic dinoflagellate Symbiodinium and their growth and host specificity. J Phycol. 2013;49:447-58.

39. Bieri T, Onishi M, Xiang T, Grossman AR, Pringle JR. Relative contributions of various cellular mechanisms to loss of algae during cnidarian bleaching. PLoS ONE. 2016;11:e0152693.

40. Sunagawa S, Wilson EC, Thaler M, Smith ML, Caruso C, Pringle JR, et al. Generation and analysis of transcriptomic resources for a model system on the rise: the sea anemone Aiptasia pallida and its dinoflagellate endosymbiont. BMC Genomics. 2009;10:258.

41. Cziesielski MJ, Liew YJ, Cui G, Schmidt-Roach S, Campana S, Marondedze C, et al. Multi-omics analysis of thermal stress response in a zooxanthellate cnidarian reveals the importance of associating with thermotolerant symbionts. Proc Biol Sci. 2018; 285:20172654.

42. Belda-Baillie CA, Baillie BK, Maruyama T. Specificity of a model cnidarian-dinoflagellate symbiosis. Biol Bull. 2002;202: $74-85$. 
43. Rodriguez-Lanetty M, Chang S-J, Song J-I. Specificity of two temperate dinoflagellate-anthozoan associations from the northwestern Pacific Ocean. Mar Biol. 2003;143:1193-9.

44. Cziesielski MJ, Schmidt-Roach S, Aranda M. The past, present, and future of coral heat stress studies. Ecol Evolution. 2019;9: 10055-66.

45. Lehnert EM, Mouchka ME, Burriesci MS, Gallo ND, Schwarz JA, Pringle JR. Extensive differences in gene expression between symbiotic and aposymbiotic cnidarians. G3 (Bethesda). 2014;4:277-95.

46. Gegner HM, Ziegler M, Rädecker N, Buitrago-López C, Aranda M, Voolstra CR. High salinity conveys thermotolerance in the coral model Aiptasia. Biol Open. 2017;6:1943.

47. Cui G, Liew YJ, Li Y, Kharbatia N, Zahran NI, Emwas A-H, et al. Host-dependent nitrogen recycling as a mechanism of symbiont control in Aiptasia. PLoS Genet. 2019;15:e1008189.

48. Röthig T, Costa RM, Simona F, Baumgarten S, Torres AF, Radhakrishnan A, et al. Distinct bacterial communities associated with the coral model aiptasia in aposymbiotic and symbiotic states with symbiodinium. Front Mar Sci. 2016;3:234.

49. Matthews JL, Sproles AE, Oakley CA, Grossman AR, Weis VM, Davy SK. Menthol-induced bleaching rapidly and effectively provides experimental aposymbiotic sea anemones (Aiptasia sp.) for symbiosis investigations. J Exp Biol. 2016;219:306.

50. Hume BCC, Ziegler M, Poulain J, Pochon X, Romac S, Boissin E, et al. An improved primer set and amplification protocol with increased specificity and sensitivity targeting the Symbiodinium ITS2 region. PeerJ. 2018;6:e4816.

51. Hume BCC, Smith EG, Ziegler M, Warrington HJM, Burt JA, LaJeunesse TC, et al. SymPortal: a novel analytical framework and platform for coral algal symbiont next-generation sequencing ITS2 profiling. Mol Ecol Resour. 2019;19:1063-80.

52. $\mathrm{R}$ Core Team. R: A language and environment for statistical computing. Vienna, Austria: R foundation for statistical computing; 2018.

53. Oksanen J, Blanchet FG, Friendly M, Kindt R, Legendre P, McGlinn D, et al. Community Ecology Package. 2019.

54. Anderson MJ. A new method for non-parametric multivariate analysis of variance. Austral Ecol. 2001;26:32-46.

55. Anderson MJ. Permutational multivariate analysis of variance (PERMANOVA). Wiley StatsRef: Statistics Reference Online. American Cancer Society; 2017. p. 1-15.

56. Anderson MJ, Willis TJ. Canonical analysis of principal coordinates: a useful method of constrained ordination for ecology. Ecology. 2003;84:511-25.

57. Love MI, Huber W, Anders S. Moderated estimation of fold change and dispersion for RNA-seq data with DESeq2. Genome Biol. 2014;15:550.

58. IBM SPSS Statistics (Version 22.0). Armonk, NY, USA: IBM Corporation; 2013.

59. Robison JD, Warner ME. Differential impacts of photoacclimation and thermal stress on the photobiology of four different phylotypes of Symbiodinium (Pyrrhophyta). J Phycol. 2006;42:568-79.

60. McGinty ES, Pieczonka J, Mydlarz LD. Variations in reactive oxygen release and antioxidant activity in multiple Symbiodinium types in response to elevated temperature. Microb Ecol. 2012;64:1000-7.

61. Grégoire V, Schmacka F, Coffroth MA, Karsten U. Photophysiological and thermal tolerance of various genotypes of the coral endosymbiont Symbiodinium sp. (Dinophyceae). J Appl Phycol. 2017;29:1893-905.

62. Lesser MP. Phylogenetic signature of light and thermal stress for the endosymbiotic dinoflagellates of corals (Family Symbiodiniaceae). Limnol Oceanogr. 2019;64:1852-63.

63. Hambleton EA, Guse A, Pringle JR. Similar specificities of symbiont uptake by adults and larvae in an anemone model system for coral biology. J Exp Biol. 2014;217:1613.
64. Wolfowicz I, Baumgarten S, Voss PA, Hambleton EA, Voolstra CR, Hatta M, et al. Aiptasia sp. larvae as a model to reveal mechanisms of symbiont selection in cnidarians. Sci Rep. 2016;6:32366.

65. Thornhill DJ, Xiang Y, Pettay DT, Zhong M, Santos SR. Population genetic data of a model symbiotic cnidarian system reveal remarkable symbiotic specificity and vectored introductions across ocean basins. Mol Ecol. 2013;22:4499-515.

66. Little AF, van Oppen MJH, Willis BL. Flexibility in algal endosymbioses shapes growth in reef corals. Science. 2004;304:1492.

67. Pettay DT, Wham DC, Smith RT, Iglesias-Prieto R, LaJeunesse TC. Microbial invasion of the Caribbean by an Indo-Pacific coral zooxanthella. Proc Natl Acad Sci USA. 2015;112:7513.

68. Baums IB, Devlin-Durante MK, LaJeunesse TC. New insights into the dynamics between reef corals and their associated dinoflagellate endosymbionts from population genetic studies. Mol Ecol. 2014;23:4203-15.

69. Nyamukondiwa C, Terblanche JS. Thermal tolerance in adult Mediterranean and Natal fruit flies (Ceratitis capitata and Ceratitis rosa): effects of age, gender and feeding status. J Therm Biol. 2009;34:406-14.

70. Dowd WW, King FA, Denny MW. Thermal variation, thermal extremes and the physiological performance of individuals. J Exp Biol. 2015;218:1956.

71. Chidawanyika F, Nyamukondiwa C, Strathie L, Fischer K. Effects of thermal regimes, starvation and age on heat tolerance of the Parthenium Beetle Zygogramma bicolorata (Coleoptera: Chrysomelidae) following dynamic and static protocols. PLoS ONE. 2017;12:e169371.

72. Hoadley KD, Lewis AM, Wham DC, Pettay DT, Grasso C, Smith R, et al. Host-symbiont combinations dictate the photo-physiological response of reef-building corals to thermal stress. Sci Rep. 2019;9: 9985.

73. Rädecker N, Raina J-B, Pernice M, Perna G, Guagliardo P, Kilburn MR, et al. Using aiptasia as a model to study metabolic interactions in cnidarian-symbiodinium symbioses. Front Physiol. 2018;9:214-214.

74. Osman EO, Smith DJ, Ziegler M, Kürten B, Conrad C, El-Haddad $\mathrm{KM}$, et al. Thermal refugia against coral bleaching throughout the northern Red Sea. Glob Change Biol. 2018;24:e474-84.

75. Berumen ML, Voolstra CR, Daffonchio D, Agusti S, Aranda M, Irigoien $\mathrm{X}$, et al. The Red Sea: environmental gradients shape a natural laboratory in a nascent ocean. In: Voolstra CR, Berumen ML, editors. Coral Reefs of the Red Sea. Cham: Springer International Publishing; 2019. p. 1-10.

76. Hawkins TD, Hagemeyer JCG, Hoadley KD, Marsh AG, Warner ME. Partitioning of respiration in an animal-algal symbiosis: implications for different aerobic capacity between Symbiodinium spp. Front Physiol. 2016;7:128.

77. Hoadley KD, Rollison D, Pettay DT, Warner ME. Differential carbon utilization and asexual reproduction under elevated $\mathrm{pCO} 2$ conditions in the model anemone, Exaiptasia pallida, hosting different symbionts. Limnol Oceanogr. 2015;60:2108-20.

78. Borell EM, Yuliantri AR, Bischof K, Richter C. The effect of heterotrophy on photosynthesis and tissue composition of two scleractinian corals under elevated temperature. J Exp Mar Biol Ecol. 2008;364:116-23.

79. Ferrier-Pagès C, Rottier C, Beraud E, Levy O. Experimental assessment of the feeding effort of three scleractinian coral species during a thermal stress: effect on the rates of photosynthesis. J Exp Mar Biol Ecol. 2010;390:118-24.

80. Connolly SR, Lopez-Yglesias MA, Anthony KRN. Food availability promotes rapid recovery from thermal stress in a scleractinian coral. Coral Reefs. 2012;31:951-60.

81. Lyndby NH, Holm JB, Wangpraseurt D, Grover R, Rottier C, Kühl $\mathrm{M}$, et al. Effect of feeding and thermal stress on photosynthesis, 
respiration and the carbon budget of the scleractinian coral Pocillopora damicornis. bioRxiv. 2018:378059.

82. Borell EM, Bischof K. Feeding sustains photosynthetic quantum yield of a scleractinian coral during thermal stress. Oecologia. 2008;157:593.

83. Weng L-C, Pasaribu B, Ping Lin I, Tsai C-H, Chen C-S, Jiang P-L. Nitrogen deprivation induces lipid droplet accumulation and alters fatty acid metabolism in symbiotic dinoflagellates isolated from aiptasia pulchella. Sci Rep. 2014;4:5777.

84. Fitt W, Cook C. The effects of feeding or addition of dissolved inorganic nutrients in maintaining the symbiosis between dinoflagellates and a tropical marine cnidarian. Mar Biol. 2001; 139:507-17.

85. Ferrier-Pagès C, Witting J, Tambutté E, Sebens KP. Effect of natural zooplankton feeding on the tissue and skeletal growth of the scleractinian coral Stylophora pistillata. Coral Reefs. 2003;22:229-40.

86. van der Merwe R, Röthig T, Voolstra CR, Ochsenkühn MA, Lattemann S, Amy GL. High salinity tolerance of the Red Sea coral Fungia granulosa under desalination concentrate discharge conditions: an in situ photophysiology experiment. Front Mar Sci. 2014;1:58. 